\title{
Practice Issues for Evaluation and Management of the Suicidal Left Ventricular Assist Device Patient
}

Yelena Chernyak, Lisa The, Danielle R. Henderson, Anahli Patel

\begin{abstract}
:
There is a high prevalence of depression among left ventricular assist device patients, who present with an increased risk for suicidality given access to means via the device either with nonadherence or disconnection. Suicidality via device nonadhrence/disconnection is an under-researched clinical issue, as paradoxically this life-saving procedure can also provide a method of lethal means to patients with significant mental health concerns.
\end{abstract}

A case study is used to highlight the course of an attempted suicide by ventricular assistive device nonadherence. Clinical implications and recommendations for practice include a thorough psychological evaluation pre-surgery, monitoring quality of life and coping styles before and after placement, psychological testing, outlining specific suicide protocols, psychiatric care considerations for patients with highly specialized medical devices, and related ethical concerns.

Keywords: Behavioral disciplines and activities, Health care facilities, manpower, and services, Cardiovascular System, Behavior and Behavior mechanisms, Pathological Conditions, signs and symptoms

This is the author's manuscript of the article published in final edited form as:

Chernyak, Y., Teh, L., Henderson, D. R., \& Patel, A. (2020). Practice Issues for Evaluation and Management of the Suicidal Left Ventricular Assist Device Patient. Progress in Transplantation, 30(1), 63-66. https://doi.org/10.1177/1526924819893300 


\section{Introduction}

There are over 10000 individuals in the United States living with a left ventricular assist device (LVAD) to treat end-stage heart failure. ${ }^{1}$ The prevalence of depression in end-stage heart failure is high, with estimates ranging from $11-70 \%,{ }^{2}$ while prevalence rates of depression of the general US population are estimated to be about $4.5 \% .{ }^{3}$ Living with an LVAD poses a unique set of psychological challenges, including adjustment to an implanted mechanical device and reduced independence. Chronically or terminally ill patients with access to nonviolent means, such as disconnecting and LVAD device, who experience depression are at increased risk for suicide ${ }^{4}$.

Clinical relevancy and practice issues are discussed below.

\section{Clinical Relevancy to Practice}

Mr. $\mathrm{G}$ is a 55-year old male with nonischemic cardiomyopathy, an implantable cardioverter-defibrillator (ICD), pulmonary hypertension, obesity, and obstructive sleep apnea implanted with an LVAD at a large urban medical center. Preceding his LVAD placement, he underwent a psychological evaluation, and unremarkable psychiatric history was notable for mild depression and anxiety.

Following LVAD placement, Mr. G adjusted poorly to the associated lifestyle changes, such as an intensive medical regiment, and decreased independence, and his mood deteriorated. Mr. G's caregivers, on whom he increasingly relied, were unable to adequately care for him, triggering social isolation, reduced sense of purpose, anhedonia, amotivation, fatigue, and nonspecific pain. Mr. G.'s medical team became concerned about his decline and referred him for follow-up with the in-house psychologist, who subsequently diagnosed him with major depressive disorder and generalized anxiety disorder. Mr. G underwent psychiatric treatment including psychiatric medical management and psychotherapy with limited success. 
Mr. G endorsed suicidal ideation with plan 7 months following his LVAD implantation, stating he intended to take out his LVAD batteries to stop his heart or discontinue his anticoagulant medications to cause a life-threatening clot. Mr. G was psychiatrically hospitalized for this reason on 6 separate occasions over a 2-year period. Psychiatric hospitalizations were typically housed on a medical floor due to the need to manage his LVAD device by trained personnel, which psychiatric floors lacked. Following his $5^{\text {th }}$ psychiatric hospitalization, he reported to his treating psychologist "I took my time changing the batteries [on my LVAD] and my defibrillator went off 8 times" in an active attempt to end his life. He noted the pain from the ICD shocks was his only barrier to completing suicide. As a result of continued depression and hopelessness, Mr. G initiated intentional nonadherence with his anticoagulation regiment and restarted smoking cigarettes in an attempt to increase the likelihood of ending his life. When he was subsequently psychiatrically admitted, his INR was significantly sub-therapeutic, confirming this follow-through of his threat of intentional nonadherence with his anticoagulation regimen.

At present, the patient continues to be followed by the advanced heart failure clinic as well as a community based mental health center for psychiatric care. His mood and medical status are closely monitored for instability; however, he does not currently meet criteria for another psychiatric or medical hospitalization.

Practice Issues: Gaps in Current Practice

While suicide completion is obviously of paramount concern, ongoing suicidality and suicide attempts can also be devastating due to psychological and physiological sequelae in LVAD patients. Depression and related suicidality can impact successful recovery from surgery, as well as dysregulation of the autonomic and parasympathetic nervous systems affecting cardiovascular functioning. ${ }^{2}$ 


\section{Pre-surgical Psychosocial Evaluation}

To address the potential for suicidality in LVAD patients, the medical team must include mental health expertise throughout the work-up, placement, and follow-up process. While many patients undergoing an LVAD implantation already a complete a psychological and/or psychosocial evaluation, the literature does not provide firm guidelines on evaluations, surgical recommendations, and interventions for depression and suicidality in LVAD patients. Consensus-based recommendations indicate that uncontrolled severe psychiatric illness, substance abuse, or active suicidality are high risk and predict premature morbidity and mortality among LVAD patients..$^{5}$ Advanced heart failure teams should include mental health professionals such as a psychologists, psychiatrists, and medical social workers to help assess LVAD candidates prior to implantation, monitor their course, and provide intervention when necessary for psychiatric or psychosocial difficulties. To facilitate this process, many validated brief measures may be used by the medical team for evaluation of depression/suicidality and psychiatric wellbeing, summarized in Table 1 from a common reference text. ${ }^{6}$

In parallel to the work-up for solid organ transplant, it might be appropriate to treat the LVAD as a prosthetic organ and evaluate the patient's understanding of the impact of the device, required lifestyle changes, social and functional limitations, and maintenance requirements. Reframing LVAD treatment as a trade-off for a subset of medical problems (ie heart failure), for a new subset (eg coagulation therapy, intensive self-monitoring), instead of curative intervention, may help mitigate expectations and set patients up for the long-term emotional success.

\section{Suicidality Management in VAD Patients}

For medical teams to successfully manage suicidality, the team must understand the risk factors for suicidality and elements leading to a suicide attempt. The majority of suicide attempts 
occur in patients suffering from depression, characterized by low mood, anhedonia, hopelessness, and worthlessness. Additionally, risk factors of suicidality include limited social support, access to means, and medical illness, the last notably present in many LVAD recipients. In addition to a psychological distress, there is a threshold a patient must psychologically overcome to proceed with the suicide attempt. This threshold is lower for soft methods (ie inaction, such as medication nonadherence) compared to hard methods (eg shooting oneself). ${ }^{7}$ As LVAD implantation may introduce easily accessible soft means to suicide, patients should be monitored for signs of depression and associated risk factors routinely. Open communication and rapport in the patient-doctor relationship are also protective factors. Appropriate psychiatric referrals, including a combination of medication management and counseling is the gold standard of care, but access can be limited, often necessitating the assistance of non-psychiatrist physicians in prescribing psychiatric medications. Further education of the treatment team can boost team skill and confidence in handling suicidality. Finally, pre-surgical, post-implantation, and ongoing annual psychological evaluations can help monitor LVAD patients for early risk factors and provide early intervention to manage depression and suicidality.

In situations where psychiatric hospitalization is indicated, such as imminent threat or actual suicide attempt, many inpatient units may not be equipped to properly evaluate or admit a patient with an LVAD. An attempt characterized by medical nonadherence with LVAD, such as failing to change batteries, may inadvertently be construed as accidental by untrained professionals. Moreover, many inpatient units do not have personnel that are trained in managing LVADs, rendering them unable to take medical responsibility of an LVAD patient that would otherwise be admitted. System wide education and policy change may be necessary to aid patients with highly specialized medical and mechanical needs obtain high quality psychiatric 
care. In the meantime, the medical team will need to collaborate on intensive management of psychiatrically ill patients with LVADs, requiring a strong multidisciplinary team for optimal outcomes, a proposed team illustrated in Figure 1.

\section{Ethical Issues in Suicidality}

In patients with LVADs, suicidality must be differentiated from terminal patients who do not wish to continue medical treatment due to poor quality of life or suffering. For most patients, a LVAD is a necessary life-saving treatment for end-stage heart failure. Swetz and colleagues argue that patients have a right to refuse treatment, viewing LVAD disconnection as a permissible choice in some instances. ${ }^{8}$ In such cases, appropriate psychiatric evaluation is necessary to differentiate the intent of this request as euthanasia via ceasing life-prolonging medical intervention from reversible psychiatric illness such as depression. Complete consensus with the entire medical team, patient, and the patient's family should be obtained. In patients with poor quality of life, collaboration of the LVAD team with hospice, palliative care, and or ethics consultants may be appropriate to help relieve suffering and fulfill the patient's wishes. ${ }^{9}$

\section{Summary and Implications for Practice}

Suicide by LVAD disconnection has been documented in the literature. ${ }^{7}$ Ventricular assistive devices provide access to lethal means as individuals depend on their implanted lifesaving device. Ending ones' life can be immediate and passive if they do not adhere closely to a strict treatment regimen. Although LVAD implantation itself has been associated with reduced depression and improved quality of 1 ife ${ }^{10}$ some patients may adjust poorly and their worsening depression combined with easy access to passive lethal means may increase their risk for suicide. Thorough psychological evaluation, routine follow-up, and targeted interventions for depressed or suicidal patients are a critical part of competent care for LVAD patients. Practitioners should 
also consider the ethical concerns of potentially giving patients the means to end their life (via a mechanical device physically outside of the body) versus providing a life-saving intervention. 
References

1. J.K. Kirklin, D.C. Naftel, F.D. Pagani, et al. Sixth INTERMACS annual report: a 10,000patient database. J Heart Lung Transplant, 33 (2014), pp. 555-564.

2. Newhouse A \& Jiang W. Heart Failure and Depression. Heart Failure Clinics 2014; 10(2):295-304.

3. National Institute of Mental Health, February 2019. Accessed Sep 20, 2019. Found at https://www.nimh.nih.gov/health/statistics/major-depression.shtml.

4. Qin P, Hawton K, Mortensen PB, \& Webb R. Combined effects of physical illness and comorbid psychiatric disorder on the risk of suicide in a national population study. The Brit J Psychiatry. 2014; 204(6): 430-435.

5. Dew MA, DiMartini AF, Dobbels, F. et. al. The 2018 ISHLT/APM/AST/ICCAC/STSW recommendations for the psychosocial evaluation of adult cardiothoracic transplant candidates and candidates for long-term mechanical circulatory support. J of Heart and Lung Transplantation 2018;37(7):803-823.

6. McDowell, I. Measuring Health: A guide to rating scales and questionnaires. 3rd edition ed. Oxford University Press; 2006.

7. Tigges-Limmer K, Schonbrodt M, Roefe D, Arusoglu L, Morshuis M, Gummert JF. Suicide after ventricular assist device implementation. J Heart Lung Transplant 2010; 29:692-4.

8. Mueller PS, Swetz KM, Freeman MR, et. al. Ethical analysis of withdrawing ventricular assist device support. Mayo Clinic Proc. 2010;85(9):791-797.

9. Swetz KM, Freeman MR, AbouEzzeddine OF, et. al. Palliative medicine consultation for preparedness planning in patients receiving left ventricular assist devices as destination therapy. Mayo Clinic Proc. 2011;86(6):493-500.

10. Reynard AK, Butler RS, McKee MG, et al. Frequency of Depression and Anxiety Before and After Insertion of a Continuous Flow Left Ventricular Assist Device. Am J of Cardiology. 2014;114(3):433-440. 
Table 1. Common Measures Used in Psychological Evaluations for Depression/Suicidalityand Psychiatric Wellbeing 6

\begin{tabular}{|l|l}
\hline Construct & Measure Name \\
\hline Depression/Suicidality & Beck Depression Inventory \\
\hline & Hospital Anxiety and Depression Scale \\
\hline Coping and Health Status & Patient Health Questionnaire Scale \\
\hline & General Health Questionnaire \\
\hline & Short Form 36 Health Survey \\
\hline Personality & Minnesota Living with Heart Failure Questionnaire \\
\hline & Minnesota Multiphasic Personality Inventory \\
\hline Quality of Life & Personality Assessment Inventory \\
\hline & \\
\hline & World Health Organization Quality of Life Scale \\
\hline Social Support & Quality of Life Scale \\
\hline & $\begin{array}{l}\text { The Duke-University of North Carolina Functional Social } \\
\text { Support Questionnaire }\end{array}$ \\
\hline
\end{tabular}


Figure 1. Medical Team's Role in Management of Psychiatric Illness/Suicidality in an LVAD Patient

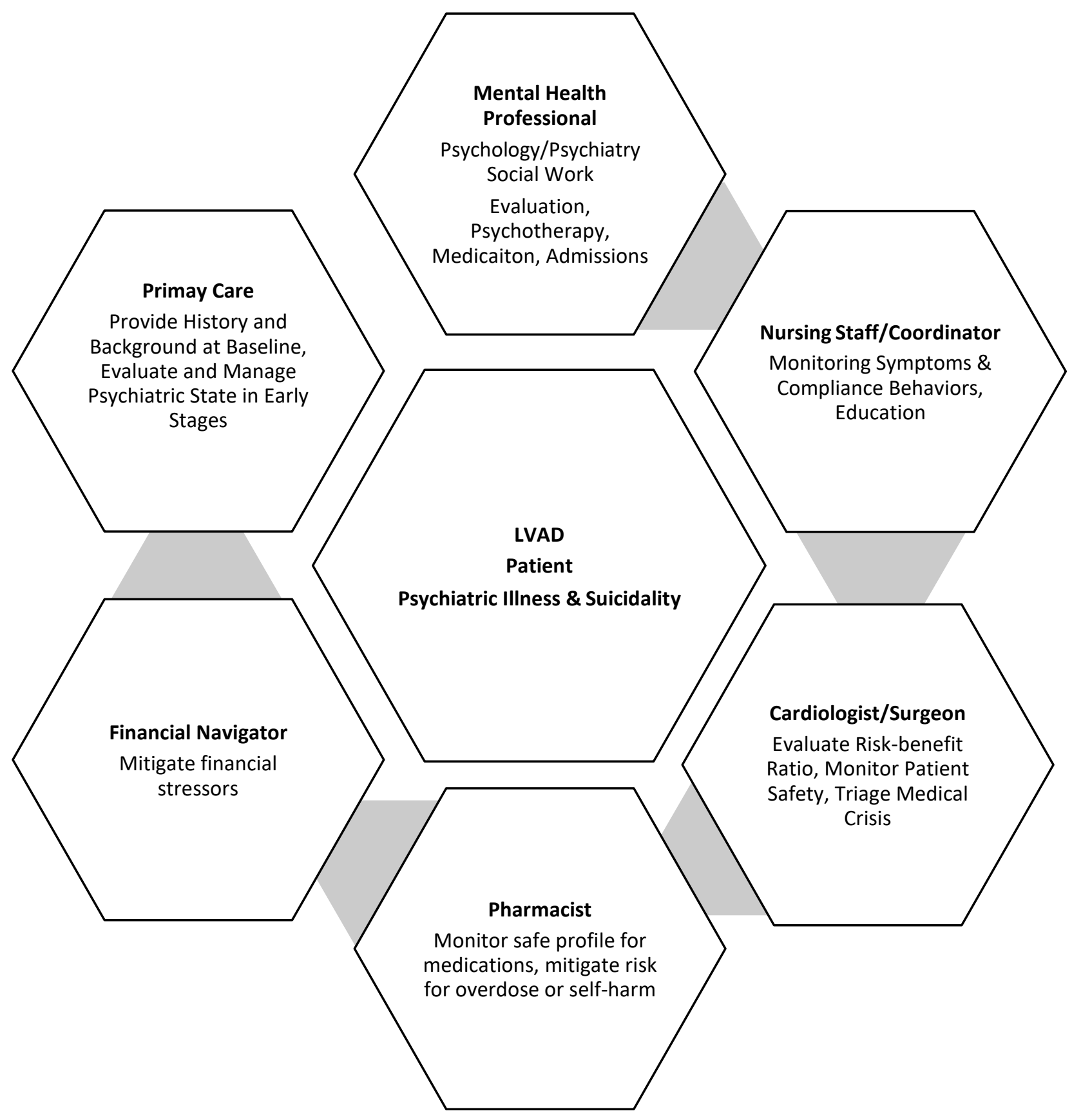

